\title{
ERRATUM
}

Dana Davis

\section{Adaptation to environmental pH in Candida albicans and its relation to pathogenesis}

Published online: 7 August 2003

(C) Springer-Verlag 2003

\section{Current Genetics (2003) \\ DOI 10.1007/s00294-003-0415-2}

In the section "Rim101p processing in C. albicans" 2 nd paragraph, the sentence "However, several issues need to be resolved, including the fact that PacC is localized to the nucleus at acidic $\mathrm{pH}$ and in the presence of upstream pathway mutants." should have read "However, several issues need to be resolved, including the fact that $\mathrm{PacC}$ is not excluded from the nucleus at acidic $\mathrm{pH}$ and in presence of upstream pathway mutants."

Also, these two references were omitted from the manuscript.

The reference Diez E et al. (2002) Activation of the Aspergillus PacC zinc finger transcription factor requires two proteolytic steps. Embo J 21:1350-1359 was omitted from the section "Rim101p processing in C. albicans" first paragraph. "In A. nidulans, processing occurs in two steps, the first which gives rise to an $\sim 500$ residue product similar to that seen in $S$. cerevisiae and a second processing event that occurs distal to the zinc finger domain and yields an $\sim 250$ residues active product (Mingot et al. 1999; Espeso et al. 2000; Diez et al. 2002)."

The reference Espeso EA, Arst HN, Jr. (2000) On the mechanism by which alkaline $\mathrm{pH}$ prevents expression of an acid-expressed gene. Mol Cell Biol 20:3355-3363 was omitted from the section "Rim101p, activator or repressor?" first paragraph. "A. nidulans PacC binds to the consensus sequence GCCAAG with high affinity and GCCAGG with lower affinity and it can act as an activator and a repressor at these sites (Tilburn et al. 1995; Espeso and Penalva 1996; Espeso et al 1997; Espeso and Arst 2000)."
The online version of the original article can be found at http:// dx.doi.org/10.1007/s00294-003-0415-2

\section{Davis}

Department of Microbiology,

University of Minnesota,

1360 Mayo Building MMC196,

Minneapolis, MN 55455, USA

E-mail: dadavis@umn.edu

Tel.: + 1-612-6241912

Fax: + 1-612-6260623 